\title{
Avaliando a Percepção de Inovação e Aceitação de uma Ferramenta de Suporte à Educação 4.0
}

\author{
Katyeudo Karlos de S. Oliveira ${ }^{1}$, Maria Lydia Fioravanti', Myke Morais de \\ Oliveira $^{1}$, Ellen Francine Barbosa ${ }^{1}$, Ricardo André C. de Souza ${ }^{2}$ \\ ${ }^{1}$ Universidade de São Paulo (USP) - São Carlos, SP, Brasil \\ ${ }^{2}$ Universidade Federal Rural de Pernambuco (UFRPE) - Recife, PE, Brasil \\ \{karlos_oliveira, mlfioravanti, mykeoliveira\}dusp.br, \\ francinedicmc.usp.br, ricardo.souza@ufrpe.br
}

Resumo. As escolas devem formar estudantes capazes de lidar com profissões e tecnologias que ainda estão por vir. A Educação 4.0 é um paradigma que está impulsionando o novo ambiente educacional por meio do ensino $e$ desenvolvimento de habilidades relevantes e tecnologias digitais. Para tanto, os professores devem ser capacitados para o uso de tais tecnologias digitais no ensino. Neste cenário, foi realizado um estudo de caso com professores sobre uma ferramenta de software de suporte à Educação 4.0, visando medir a percepção de inovação e aceitação da ferramenta. A percepção de inovação dos professores foi positiva, principalmente, como software habilitador da resolução criativa de problemas e da transformação do conhecimento. Os professores concordaram com os indicadores de facilidade de uso e de utilidade de uso percebidos.

\begin{abstract}
Schools must train students to be capable of dealing with professions and technologies that are yet to come. Education 4.0 is a paradigm that is leveraging the new educational environment through teaching and developing relevant skills and digital technologies. Therefore, teachers must be trained in the use of such digital technologies in teaching. In this scenario, a case study was carried out with teachers about a software tool to support Education 4.0, aiming to measure the innovation perception and tool acceptance. Teachers' innovation perception was positive, mainly as enabling software for creative problem solving and knowledge transformation. Teachers agreed with the indicators of ease of use and perceived usefulness of use.
\end{abstract}

\section{Introdução}

De acordo com o World Economic Forum [WEF 2016], algumas profissões atuais não existiam na década passada, tais como: arquiteto de Big Data, cientista de dados/cloud computing, analista de marketing digital, analista de segurança da informação, entre outros. Porém, os sistemas educacionais ainda se orientam por meio de modelos utilizados há décadas e, à medida que o mundo entra na $4^{\mathrm{a}}$ Revolução Industrial, é importante que a sociedade tenha conhecimento da real necessidade de mudanças abrangentes na educação.

As escolas devem formar estudantes capazes de lidar com profissões e tecnologias que ainda estão por vir [OECD 2018b]. Segundo Partovi (2018), a sociedade tem o desafio de redirecionar a educação para acompanhar a evolução das habilidades necessárias aos estudantes para resolver problemas e obter sucesso. A introdução de 
novos conceitos no currículo como, por exemplo, o pensamento computacional, visa preparar os estudantes com competências como a criatividade, resolução de problemas, ética e colaboração.

A Educação 4.0 é um paradigma que está impulsionando o novo ambiente educacional a partir do uso de habilidades relevantes, baseando-se na necessidade de aprimorar e requalificar, desaprender e reaprender [WEF 2020] [Hong e Ma 2020]. Para tanto, os professores devem ser capacitados para o uso de tecnologias digitais no ensino [Schleicher 2016; De Sousa Oliveira et al. 2019]. Segundo [De Sousa Oliveira e de Souza 2020], os professores necessitam ser treinados com competências digitais, com o uso de tecnologias, pedagogias inovadoras e a adoção de mentalidade e atitudes digitais.

Além disso, as mudanças tecnológicas e a maior disponibilidade de recursos digitais estão produzindo novos caminhos no processo de ensino e aprendizagem [OECD 2018b]. De acordo com Barbosa e Souza (2021), as tecnologias digitais, em especial as ferramentas de software, são recursos que, se utilizados de modo adequado, podem habilitar, transformar ou dar apoio à inovação na educação. Assim, se faz necessário um suporte para que os professores consigam uma base para adquirir novas habilidades e aprender como ensiná-las [OECD 2018b].

Considerando o contexto discutido anteriormente, foi proposto o método TADEO [Oliveira e de Souza 2021], acrônimo de Transformação Digital da Educação, cujo objetivo é orientar o desenho e aplicação de experiências de ensino e aprendizagem a partir de conjuntos de habilitadores da transformação digital da educação, visando alcançar objetivos da Educação 4.0, por meio do uso holístico dos habilitadores humanos, tecnológicos, pedagógicos e organizacionais, no desenvolvimento de experiências de ensino e aprendizagem.

Além disso, o método TADEO propõe o uso de uma ferramenta de software como suporte tecnológico, visando contribuir com o alcance de propósitos da Educação 4.0, como estimular, exercitar e equipar os estudantes com as habilidades apontadas como necessárias para o aprendizado e trabalho do século XXI.

Considerando o cenário mencionado, neste artigo é apresentado um estudo de caso com professores sobre a ferramenta de software de suporte à Educação 4.0, considerando os seguintes aspectos: (i) percepção de inovação; e (ii) aceitação da ferramenta. Para medir a percepção de inovação, foi utilizada a abordagem proposta por Barbosa e Souza (2021). No que se refere à aceitação da ferramenta pelos usuários, foi aplicado o Modelo de Aceitação de Tecnologia - Technology Acceptance Model (TAM) [Davis 1989].

O restante deste artigo está organizado da seguinte forma. Na Seção 2, a ferramenta de suporte à Educação 4.0 é descrita. Na Seção 3, o método de pesquisa aplicado é discutido. Os resultados são apresentados e discutidos na Seção 4. Por fim, as conclusões e trabalhos futuros são apresentados na Seção 5.

\section{Ferramenta de Suporte à Educação 4.0}

A ferramenta ${ }^{1}$ foi desenvolvida para dar suporte ao método TADEO. É uma ferramenta web, com as seguintes funcionalidades principais: (i) painel interativo com os

\footnotetext{
${ }^{1}$ http://tadeo.com.br
} 
habilitadores da transformação digital na educação; (ii) construção de planos de ensino, por meio da seleção do conjunto de habilitadores, o objetivo do ensino, a duração das atividades, os materiais necessários e o método avaliativo que será utilizado; (iii) repositório digital dos produtos de trabalho produzidos pelos estudantes nas experiências de ensino e aprendizagem; e (iv) dashboards com o resultado das autoavaliações e avaliações entre pares realizadas pelos estudantes.

A ferramenta disponibiliza algumas experiências já aplicadas, sendo possível visualizar as apresentações produzidas pelos estudantes ou fazer o download dos planos de ensino produzidos pelos professores. Ainda, contém um dashboard dos habilitadores da Educação 4.0, sendo apresentada uma breve descrição de cada um, com base na análise de documentos de organizações internacionais de referência. Neste cenário, os habilitadores dão suporte, direcionam iniciativas de Educação 4.0. Conforme apresentado por [De Sousa Oliveira e de Souza 2020], tais habilitadores podem ser agrupados nas seguintes categorias: Tecnológico, Organizacional, Competência digital docente, Soft skill discente, Hard skill discente e Pedagogia.

A principal contribuição da ferramenta se refere à possibilidade de especificar e produzir planos de ensino, contendo: (i) o que o estudante poderá aprender; (ii) a duração para a execução do plano; (iii) as disciplinas envolvidas; (iv) o material necessário; e (v) o método avaliativo que será utilizado com os estudantes. Além disso, o professor poderá encontrar os habilitadores para especificar quais e como cada um será utilizado.

\section{Estudo de Caso}

Para a condução deste estudo, foram seguidas as diretrizes propostas por Runeson e Höst (2009) para projetar e relatar um estudo de caso. A seguir, são apresentados os detalhes do protocolo.

\subsection{Objetivo, Questão de Pesquisa e Seleção de Sujeitos}

Este estudo de caso foi planejado visando investigar as seguintes questões de pesquisa $(\mathrm{QP})$ :

- QP1: Qual a percepção de inovação educacional dos professores em relação à ferramenta de suporte à Educação 4.0?

- QP2: Qual o grau de aceitação dos professores em relação à ferramenta de suporte à Educação 4.0?

As questões de pesquisa são abordadas por meio de um estudo de caso, visto que tal metodologia é apropriada quando os pesquisadores necessitam realizar uma análise holística de um fenômeno em seu contexto de vida real. Além disso, um estudo de caso dá a possibilidade de avaliar questões de uma perspectiva prática ao invés de teórica [Yin 2009]. Considerando tal definição e as orientações de Runeson e Höst (2009), este artigo é classificado como um estudo de caso explicativo, ou seja, avalia como e por que uma nova intervenção funcionou [Yin 1999], uma vez que tem como objetivo avaliar a percepção de inovação educacional e grau de aceitação dos professores ao utilizar a ferramenta de software do método TADEO.

Para participar do estudo, o professor tinha que estar atuando em alguma disciplina. Foram enviados 18 convites, não obtendo retorno de 2 dos convidados. 
Ressalta-se que a participação foi voluntária, deixando claro que participariam do estudo sem serem beneficiados de nenhuma forma. Portanto, neste trabalho, um caso (avaliação de uma ferramenta de software) foi realizado com 16 professores, com diferentes níveis de formação acadêmica, área e disciplina de atuação, e tempo de experiência profissional.

\subsection{Tarefas}

As tarefas do estudo foram organizadas em quatro fases, da seguinte forma:

- Fase 1 - Preparação: Os pesquisadores apresentaram aos participantes os objetivos gerais e condições do estudo. De acordo, o participante preenche o termo de consentimento online e responde ao questionário de caracterização, que coleta informações acerca da sua formação acadêmica, tempo de experiência profissional e disciplina de atuação;

- Fase 2 - Treinamento: Foi disponibilizado um treinamento sobre os conceitos de Educação 4.0, habilitadores, habilidades do século XXI e as funcionalidades da ferramenta de software. Em seguida, foram disponibilizados dois vídeos de experiências já executadas no contexto da Educação 4.0. Essa fase visou treinar os professores com os conhecimentos necessários e mostrar como pode ser possível utilizá-los no ambiente educacional;

- Fase 3 - Construção de planos de ensino: Os pesquisadores disponibilizam a ferramenta para que os professores construam um plano de ensino no contexto da Educação 4.0.

- Fase 4 - Avaliação dos professores: Em seguida, todos os professores preencheram um formulário para medir o nível de percepção de inovação educacional e aceitação da ferramenta manuseada.

\subsection{Coleta de Dados}

A coleta de dados foi organizada de modo a responder a ambas as questões de pesquisa propostas.

Visando responder à QP1, que se refere à percepção de inovação educacional, foi utilizada uma abordagem específica para produtos de software educacional, proposta por Barbosa e Souza (2021). Tal abordagem, denominada DEISE (acrônimo de DEterminantes e Indicadores para Softwares de suporte à Educação), visa orientar, além da construção, a avaliação de produtos de software de suporte à educação. A abordagem é baseada em fatores determinantes e indicadores de inovação com uma perspectiva de fomentar as habilidades e competências necessárias para os estudantes do século XXI. Segundo os autores, os determinantes levam à inovação em educação (fatores técnicos e pedagógicos considerados no desenvolvimento de software educacional) e os indicadores medem à inovação em educação (fatores que avaliam se o software de suporte à educação fomenta as habilidades e competências do século XXI). Neste contexto, a abordagem inclui um questionário utilizado para avaliar os indicadores, conforme pode ser visto na Tabela 1.

Tabela 1. Indicadores de Inovação em Educação. [Barbosa e Souza 2021]

\begin{tabular}{|c|l|}
\hline Indicador & \multicolumn{1}{|c|}{ Descrição } \\
\hline$[101]$ & Software como habilitador do aprendizado autônomo e autodirigido. \\
\hline$[102]$ & $\begin{array}{l}\text { Software como habilitador da adaptação a diferentes modos e paradigmas de } \\
\text { aprendizagem. }\end{array}$ \\
\hline
\end{tabular}




\begin{tabular}{|c|l|}
\hline$[103]$ & Software como habilitador do aprendizado personalizado. \\
\hline$[104]$ & $\begin{array}{l}\text { Software como habilitador da contribuição individual em um esforço coletivo para realização } \\
\text { de uma tarefa. }\end{array}$ \\
\hline$[105]$ & Software como habilitador do aprendizado em pares. \\
\hline$[106]$ & Software como habilitador da comunicação entre indivíduos. \\
\hline$[107]$ & Software como habilitador da comunicação criativa do aprendizado. \\
\hline$[108]$ & Software como habilitador da resolução criativa de problemas. \\
\hline$[109]$ & Software como habilitador da transformação do conhecimento. \\
\hline
\end{tabular}

Em uma perspectiva relacionada, para responder à QP2, que se refere ao grau de aceitação dos professores em relação à ferramenta foi utilizado o questionário baseado nos indicadores do modelo TAM [Davis 1989], utilizado amplamente em pesquisas para avaliar a causa de usuários aceitarem ou rejeitarem uma determinada tecnologia [Venkatesh e Bala 2008]. Os indicadores utilizados foram: Facilidade de Uso Percebida (FUP), que define o nível em que uma pessoa julga que usar uma tecnologia seria livre de esforço; e Utilidade Percebida (UP), que define o nível que uma pessoa julga que tal tecnologia pode aperfeiçoar seu desempenho no trabalho. O motivo do uso desses indicadores é que, segundo Davis (1989), tais aspectos são altamente correlacionados com a ação de aceitação da tecnologia pelo usuário. Neste contexto, os professores responderam a um questionário (Tabela 2) de escala Likert de 5 pontos, variando de (1) "discordo totalmente" a (5) "concordo totalmente", com 8 questões fechadas: 4 questões para FUP e 4 para UP.

Tabela 2. Questões para a Análise da Aceitação de Tecnologia.

\begin{tabular}{|c|l|}
\hline Indicador & \multicolumn{1}{|c|}{ Descrição } \\
\hline [FUP01] & Minha interação com a ferramenta foi clara e compreensível. \\
\hline [FUP02] & Utilizar a ferramenta não exige muito do meu esforço mental. \\
\hline [FUP03] & Considero a ferramenta fácil de utilizar. \\
\hline [FUP04] & Considero fácil usar a ferramenta para construir planos de ensino. \\
\hline [UP01] & Usar a ferramenta melhora o meu desempenho na construção de planos de ensino. \\
\hline [UP02] & $\begin{array}{l}\text { A ferramenta produz os resultados que espero de uma ferramenta de suporte ao processo de } \\
\text { ensino e aprendizagem. }\end{array}$ \\
\hline [UP03] & Usar a ferramenta melhora a minha produtividade. \\
\hline [UP04] & Usar a ferramenta é importante para o processo de ensino e aprendizagem da Educação 4.0. \\
\hline
\end{tabular}

\subsection{Unidades de análise e procedimento de análise}

Os dados coletados foram avaliados por meio das métricas definidas na Seção 3.4, ou seja, a percepção de inovação educacional e a aceitação de tecnologia por cada professor com relação à ferramenta utilizada no estudo de caso. Os dados foram sintetizados por meio de análise estatística, conforme apresentado na Seção 4.

\subsection{Ameaças à validade}

Uma potencial ameaça à validade interna deste estudo diz respeito à validade do construto, neste caso, se os questionários possuírem perguntas ambíguas e formuladas de modo inapropriado. Para mitigar esta ameaça, foram adotados questionários já validados pela comunidade acadêmica. Outra possível limitação dos questionários é a dúvida quanto à resposta sincera dos participantes. Para mitigar esta ameaça, foi esclarecido aos professores que a participação seria voluntária e que nenhum dado coletado levaria à sua identificação pessoal. Porém, esta ameaça nunca pode ser totalmente excluída.

Quanto à ameaça à validade externa, uma potencial ameaça é a quantidade de voluntários deste estudo. Assim, não é possível generalizar os resultados obtidos para a 
população-alvo de onde foi retirada a sua amostra. Para mitigar esta ameaça, foram incluídos professores de diversas áreas de atuação e formação acadêmica.

\section{Resultados e Discussão}

\subsection{Caracterização dos Sujeitos}

Foram coletadas informações de 16 professores. Inicialmente, foi realizada a caracterização dos professores participantes do estudo. A Figura 1(a) mostra o quantitativo da formação acadêmica dos professores, com destaque para "Especialização" e "Mestrado" com 6 participantes cada. Em seguida, foi perguntado qual a área de atuação dos professores. A Figura 1(b) mostra o quantitativo, com maior destaque para "Ensino Fundamental" e "Ensino Superior" com 6 participantes cada.

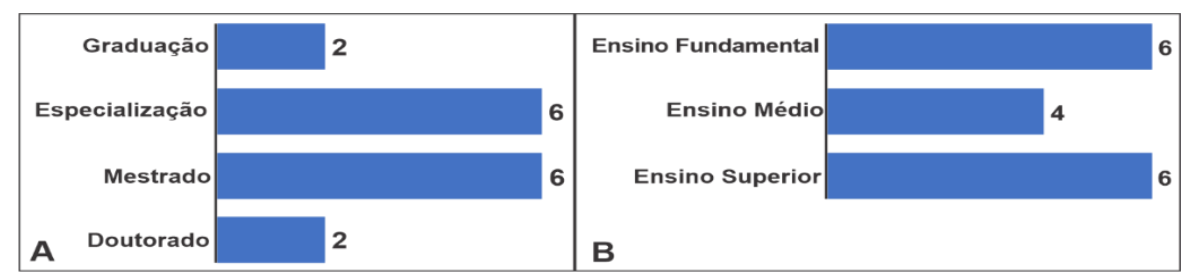

Figura 1. (a) Formação acadêmica. (b) Área de atuação.

Também, foi perguntado qual a principal disciplina de atuação dos professores. A Figura 2(a) mostra o quantitativo, com maior destaque para "Tecnologia" com 5 participantes. Por fim, foi perguntado qual o tempo de atuação dos professores. A Figura 2(b) mostra o quantitativo, com maior destaque para "entre 3 e 5 anos" com 6 participantes.

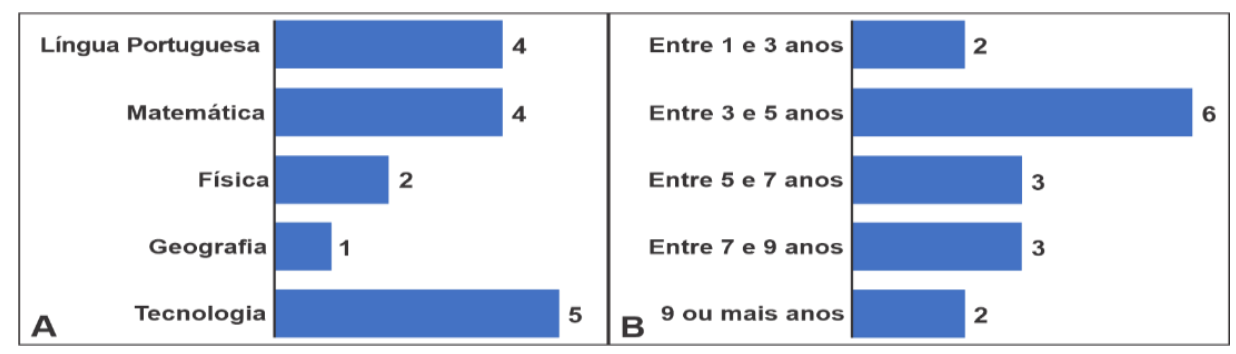

Figura 2. (a) principal disciplina de atuação. (b) Tempo de atuação.

\subsection{Percepção de Inovação Educacional dos Professores (RQ1)}

A percepção de inovação educacional dos professores em relação à ferramenta, obtida por meio deste estudo, pode ser visualizada na Tabela 3.

Tabela 3. Indicadores de Inovação em Educação dos Professores.

\begin{tabular}{|c|c|c|c|c|c|c|c|c|c|c|c|c|c|c|c|c|c|c|}
\hline Ind & \multicolumn{2}{|c|}{ [l01] } & \multicolumn{2}{|c|}{ [I02] } & \multicolumn{2}{|c|}{ [I03] } & \multicolumn{2}{|c|}{ [I04] } & \multicolumn{2}{|c|}{ [105] } & \multicolumn{2}{|c|}{ [106] } & \multicolumn{2}{|c|}{ [107] } & \multicolumn{2}{|c|}{ [108] } & \multicolumn{2}{|c|}{ [109] } \\
\hline EV & $\begin{array}{l}\mathrm{A} \\
\mathrm{V}\end{array}$ & $\begin{array}{l}A \\
S\end{array}$ & $\begin{array}{l}A \\
V\end{array}$ & $\begin{array}{l}A \\
S\end{array}$ & $\begin{array}{l}A \\
V\end{array}$ & $\begin{array}{l}A \\
S\end{array}$ & $\begin{array}{l}A \\
V\end{array}$ & $\begin{array}{l}A \\
S\end{array}$ & $\begin{array}{l}\mathrm{A} \\
\mathrm{V}\end{array}$ & $\begin{array}{l}A \\
S\end{array}$ & $\begin{array}{l}\mathrm{A} \\
\mathrm{V}\end{array}$ & $\begin{array}{l}A \\
S\end{array}$ & $\begin{array}{l}\mathrm{A} \\
\mathrm{V}\end{array}$ & $\begin{array}{l}A \\
S\end{array}$ & $\begin{array}{l}\mathrm{A} \\
\mathrm{V}\end{array}$ & $\begin{array}{l}A \\
S\end{array}$ & $\begin{array}{l}\mathrm{A} \\
\mathrm{V}\end{array}$ & $\begin{array}{l}A \\
S\end{array}$ \\
\hline EV1 & 4 & 1 & 4 & 1 & 4 & 1 & 3 & 0,5 & 3 & 0,5 & 3 & 0,5 & 4 & 1 & 4 & 1 & 4 & 1 \\
\hline EV2 & 4 & 1 & 3 & 0,5 & 3 & 0,5 & 3 & 0,5 & 3 & 0,5 & 3 & 0,5 & 3 & 0,5 & 4 & 1 & 4 & 1 \\
\hline EV3 & 4 & 1 & 4 & 1 & 4 & 1 & 4 & 1 & 4 & 1 & 3 & 0,5 & 4 & 1 & 4 & 1 & 4 & 1 \\
\hline EV4 & 3 & 0,5 & 3 & 0,5 & 3 & 0,5 & 2 & 0 & 2 & 0 & 3 & 0,5 & 3 & 0,5 & 3 & 0,5 & 4 & 1 \\
\hline EV5 & 2 & 0 & 3 & 0,5 & 3 & 0,5 & 2 & 0 & 3 & 0,5 & 4 & 1 & 4 & 1 & 4 & 1 & 4 & 1 \\
\hline EV6 & 3 & 0,5 & 4 & 1 & 3 & 0,5 & 3 & 0,5 & 3 & 0,5 & 4 & 1 & 4 & 1 & 4 & 1 & 4 & 1 \\
\hline EV7 & 3 & 0,5 & 3 & 0,5 & 3 & 0,5 & 3 & 0,5 & 3 & 0,5 & 3 & 0,5 & 3 & 0,5 & 3 & 0,5 & 4 & 1 \\
\hline EV8 & 4 & 1 & 4 & 1 & 4 & 1 & 3 & 0,5 & 2 & 0 & 3 & 0,5 & 3 & 0,5 & 3 & 0,5 & 4 & 1 \\
\hline EV9 & 3 & 0,5 & 3 & 0,5 & 3 & 0,5 & 3 & 0,5 & 3 & 0,5 & 4 & 1 & 4 & 1 & 3 & 0,5 & 3 & 0,5 \\
\hline EV10 & 4 & 1 & 4 & 1 & 3 & 0,5 & 2 & 0 & 2 & 0 & 2 & 0 & 2 & 0 & 3 & 0,5 & 3 & 0,5 \\
\hline
\end{tabular}




\begin{tabular}{|c|c|c|c|c|c|c|c|c|c|c|c|c|c|c|c|c|c|c|}
\hline EV11 & 3 & 0,5 & 3 & 0,5 & 4 & 1 & 3 & 0,5 & 2 & 0 & 3 & 0,5 & 2 & 0 & 4 & 1 & 4 & 1 \\
\hline EV12 & 4 & 1 & 3 & 0,5 & 3 & 0,5 & 3 & 0,5 & 3 & 0,5 & 3 & 0,5 & 3 & 0,5 & 3 & 0,5 & 4 & 1 \\
\hline EV13 & 4 & 1 & 4 & 1 & 3 & 0,5 & 3 & 0,5 & 3 & 0,5 & 4 & 1 & 3 & 0,5 & 4 & 1 & 4 & 1 \\
\hline EV14 & 4 & 1 & 4 & 1 & 3 & 0,5 & 3 & 0,5 & 3 & 0,5 & 4 & 1 & 3 & 0,5 & 3 & 0,5 & 4 & 1 \\
\hline EV15 & 3 & 0,5 & 3 & 0,5 & 4 & 1 & 4 & 1 & 4 & 1 & 4 & 1 & 4 & 1 & 4 & 1 & 4 & 1 \\
\hline EV16 & 2 & 0 & 3 & 0,5 & 2 & 0 & 2 & 0 & 3 & 0,5 & 3 & 0,5 & 3 & 0,5 & 3 & 0,5 & 4 & 1 \\
\hline $\begin{array}{l}\text { I2E } \\
\text { Ind }\end{array}$ & & & & & & & & & & & & & & & & & & \\
\hline
\end{tabular}

De acordo com Barbosa e Souza (2021), na análise do software educacional, é atribuída uma pontuação (assigned score - AS), associando-se a um valor (assigned value - AV), informada por um avaliador (evaluator - EV) para cada indicador de I01 a I09, onde: 1 - Não Atende; 2 - Não Sei; 3 - Atende Parcialmente; e 4 - Atende Plenamente. Caso a nota seja 1 ou 2, é atribuída a pontuação 0 no respectivo indicador. Caso a nota seja 3 é dada a pontuação 0,5 . E, caso a nota seja 4 é atribuída a pontuação 1 . Assim, o Índice de Inovação em Educação (Index to Education - I2E) é calculado pela média das pontuações atribuídas aos indicadores de inovação, portanto, o I2E varia de 0 a 1 .

O gráfico de radar (Figura 3), sintetiza os dados apresentados previamente. O objetivo é facilitar a visualização da percepção dos professores, representada pelos valores atribuídos aos indicadores de inovação dos elementos analisados em uma visão mais interna (pior resultado) para uma visão mais externa (melhor resultado).

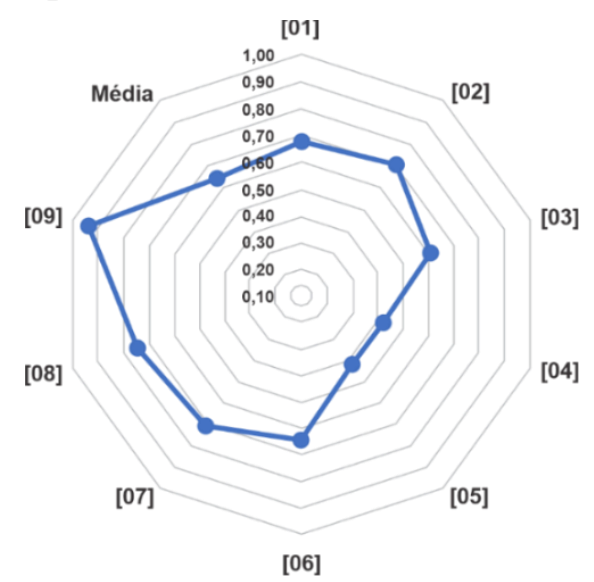

Figura 3. Gráfico de Radar dos Indicadores de Inovação em Educação.

Nota-se que o indicador I04 foi um dos indicadores que teve em média a menor avaliação pelos professores (43\%), o que significa que não foi percebida uma grande contribuição individual em um esforço coletivo para realização de uma tarefa, em particular, para a construção de planos de ensino pela ferramenta de software avaliada. $\mathrm{O}$ I05 foi um outro indicador que não foi muito bem avaliado (43\%), ou seja, a maioria dos professores não foi capaz de perceber a ferramenta como um habilitador do aprendizado em pares. O indicador com a melhor avaliação foi o I09 (93\%), sugerindo que o software tem a capacidade de ser um habilitador da transformação do conhecimento. Em seguida, outro indicador que se destacou foi o I08 (75\%), assim, percebe-se que o software pode ser um habilitador da resolução criativa de problemas.

Finalmente, considerando a primeira questão de pesquisa deste estudo, de acordo com Barbosa e Souza (2021), os resultados indicam que os professores relataram positivamente a percepção de inovação educacional da ferramenta de software, principalmente para: 
- Software como habilitador da transformação do conhecimento: possibilitar com que o usuário externalize um conhecimento adquirido ou recombinar distintas peças do conhecimento;

- Software como habilitador da resolução criativa de problemas: possibilita com que o usuário demonstre o conhecimento adquirido (internalizado) de modo criativo;

- Software como habilitador da adaptação a diferentes modos e paradigmas de aprendizagem: possibilitar com que o usuário se adapte a distintas formas de aprendizado, por exemplo, aprendizagem ativa;

- Software como habilitador do aprendizado autônomo e autodirigido: possibilita com que o usuário aprenda por si só e defina seus objetivos.

\subsection{Análise da Aceitação de Tecnologia (QP2)}

Os professores também relataram o seu grau de aceitação em relação a ferramenta de software por meio de respostas a um questionário TAM. A Figura 4 mostra a opinião dos professores do estudo em relação à facilidade de uso percebida.

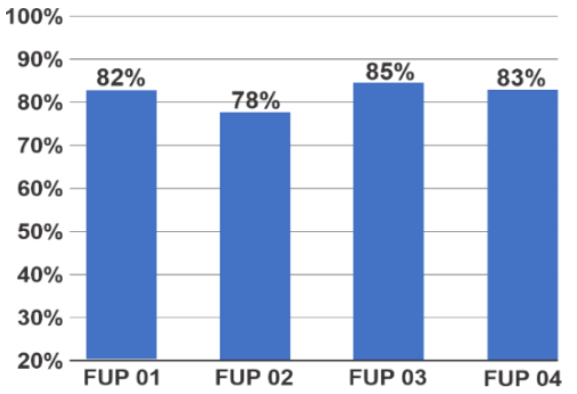

Figura 4. Facilidade de uso percebida da ferramenta.

Ao observar os dados fornecidos pelo gráfico contido na Figura 4, nota-se que os professores concordaram com as quatro sentenças do indicador de FUP, ficando com todas as médias em pelo menos $78 \%$. O indicador FUP03 foi o que recebeu a maior avaliação pelos professores $(85 \%)$, justamente o indicador com referência explícita à facilidade de uso de modo geral da ferramenta avaliada. Em seguida, o FUP04 recebeu $83 \%$ de avaliação positiva dos professores, outro indicativo da facilidade de uso da ferramenta, desta vez, sobre a construção de planos de ensino, o principal objetivo do software avaliado. Assim, nota-se, principalmente, que a ferramenta pode ser utilizada sem muito esforço para uso de modo geral e para a construção de planos de ensino.

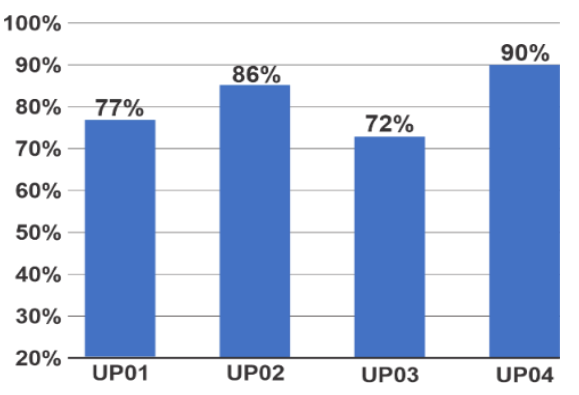

Figura 5. Utilidade percebida da ferramenta.

Na Figura 5, observa-se a opinião dos professores em relação à utilidade percebida da ferramenta de software. Em média, os professores avaliaram como positiva as quatro 
sentenças do indicador UP, ficando com todas as médias com pelo menos $72 \%$. O indicador UP04 foi o que recebeu a maior avaliação referente à utilidade da ferramenta, assim, sugerindo a sua importância para o processo de ensino e aprendizagem da Educação 4.0. Na sequência, com 86\% o UP02 foi o indicador com maior avaliação, ou seja, de acordo com os professores, a ferramenta produz os resultados esperados de uma ferramenta de suporte ao processo de ensino e aprendizagem.

Os resultados obtidos a partir do feedback dos professores apontam para facilidade e utilidade de uso da ferramenta, sendo consistente com a afirmação da OECD (2018a), que diz que as ferramentas de software possuem potencial para transformar o processo de aprendizagem, por exemplo, possibilitando um aprendizado mais aprofundado e flexível. Porém, o aprendizado necessita da qualidade do software que é disponibilizado no ambiente escolar. Sobrecarregar os professores com novas tecnologias ou produtos pode dificultar o processo de ensino. Portanto, tais ferramentas devem ser úteis ao processo de ensino e acessíveis aos professores, de forma gradativa e em momentos apropriados.

Como relatado, o uso da ferramenta possui importância para o processo de ensino e aprendizagem da Educação 4.0, alinhado com o relatório Schools of the Future do Fórum Econômico Mundial, que aponta que a Educação 4.0 exige mecanismos de aprendizado que reflitam de maneira mais próxima o futuro do trabalho e que utilizem o máximo possível as oportunidades proporcionadas pelas novas tecnologias de ensino [WEF 2020]. Desse modo, como resposta à segunda questão de pesquisa deste estudo, os resultados indicam que os professores demonstraram um elevado nível de aceitação em relação à ferramenta de suporte à Educação 4.0.

\section{Conclusão}

Este artigo apresenta um estudo de caso conduzido com professores a respeito de uma ferramenta de software de suporte à Educação 4.0. Foram estabelecidas questões de pesquisa para investigar tanto a percepção de inovação [Barbosa e Souza 2021], quanto a aceitação da ferramenta [Davis 1989] por professores. Em geral, nove indicadores de inovação educacional e oito indicadores de aceitação foram avaliados ao longo do estudo.

Como resultados referentes à primeira questão de pesquisa, os professores indicaram que, de modo geral, a ferramenta possui um alto índice de inovação educacional, sugerindo que a ferramenta pode ser utilizada principalmente na resolução criativa de problemas, na transformação do conhecimento, na adaptação a diferentes modos e paradigmas de aprendizagem e no aprendizado autônomo e autodirigido. Com relação à segunda questão de pesquisa, os professores relataram a aceitação da ferramenta, observado que os professores apontaram facilidade e utilidade de uso da ferramenta. Assim, sugerindo positivamente o uso da ferramenta para o processo de ensino na Educação 4.0, o que indica um resultado satisfatório para o estudo de caso.

Como trabalhos futuros, pretende-se replicar este estudo com um número maior de professores e áreas de atuação. Ainda, almeja-se implementar outras funcionalidades na ferramenta, por exemplo: fóruns de debate e tradução para a língua inglesa. Também, desenvolver uma Comunidade de Interesse e uma Comunidade de Prática para discussão de estratégias para a construção de um Modelo de Negócio para a ferramenta de software de suporte método TADEO. 


\section{Agradecimentos}

Os autores agradecem às agências de fomento brasileiras - Fundação de Amparo à Pesquisa do Estado de São Paulo (FAPESP) sob o processo no 2018 / 26636-2 e Coordenação de Aperfeiçoamento de Pessoal de Nível Superior - Brasil (CAPES) Código Financeiro 001.

\section{Referências}

Barbosa, R. and Souza, R. (2021). Drivers and Indicators of Innovation to Educational Software. Informatics in Education, 20(1), 1-17.

Davis, F. D. (1989). Perceived usefulness, perceived ease of use, and user acceptance of information technology. MIS quarterly, 319-340.

De Sousa Oliveira, K. K., \& de Souza, R. A. C. (2020). Habilitadores da transformação digital em direção à Educação 4.0. RENOTE, 18(1).

De Sousa Oliveira, K. K., Andrade, C. W. L., Segundo, J., Cândido, A. L., Oliveira, A., dos Santos Machado, A., and de Britto, A. C. (2019). Tecnologias na Educação Básica uma Pesquisa Ação com Professores de uma Escola Pública Municipal. In Anais do Workshop de Informática na Escola (Vol. 25, No. 1, pp. 1054-1058).

OECD. Organisation for Economic Co-operation and Development. (2018a) Teaching for the future: Effective classroom practices to transform education. $O E C D$, Publishing, Paris.

OECD. Organisation for Economic Co-operation and Development. (2018b). The future of education and skills: Education 2030. OECD Education 2030. OECD Publishing, Paris.

Oliveira, K. K. D. S., and de Souza, R. A. (2021). Digital Transformation towards Education 4.0. Informatics in Education. doi:10.15388/infedu.2022.13.

Partovi, H. (2018). "Why schools should teach the curriculum of the future, not the past". https://www.weforum.org/agenda/2018/09/why-schools-should-teach-thecurriculum-of-the-future-not-the-past. Maio.

Runeson, P. and Höst, M. (2009). Guidelines for conducting and reporting case study research in software engineering. Empirical software engineering, 14(2), 131-164.

Schleicher, A. (2016), Teaching Excellence through Professional Learning and Policy Reform: Lessons from Around the World, International Summit on the Teaching Profession, OECD Publishing, Paris.

Venkatesh, V. and Bala, H. (2008). Technology acceptance model 3 and a research agenda on interventions. Decision sciences, 39(2), 273-315.

WEF. World Economic Forum. (2016). The future of jobs: Employment, skills and workforce strategy for the fourth industrial revolution. In Global Challenge Insight Report, World Economic Forum. Cologny/Geneva, Switzerland

WEF. World Economic Forum. (2020). Schools of the Future. Defining New Models of Education for the Fourth Industrial Revolution. In Platform for Shaping the Future of the New Economy and Society. Cologny/Geneva, Switzerland.

Yin, R. K. (1999). Enhancing the quality of case studies in health services research. Health services research, 34(5 Pt 2), 1209.

Yin, R. K. (2009). "Case study research: Design and methods (Vol. 5)". sage. 